\title{
Improving SVM-Linear Predictions Using CART for Example Selection
}

\author{
João M. Moreira ${ }^{1}$, Alípio M. Jorge ${ }^{2}$, Carlos Soares ${ }^{3}$, and Jorge Freire de Sousa ${ }^{4}$ \\ ${ }^{1}$ Faculty of Engineering, University of Porto, Portugal \\ jmoreira@fe.up.pt \\ ${ }^{2}$ Faculty of Economics, LIACC, University of Porto, Portugal \\ amjorge@liacc.up.pt \\ ${ }^{3}$ Faculty of Economics, LIACC, University of Porto, Portugal \\ csoares@liacc.up.pt \\ ${ }^{4}$ Faculty of Engineering, University of Porto, Portugal \\ jfsousa@fe.up.pt
}

\begin{abstract}
This paper describes the study on example selection in regression problems using $\mu$-SVM (Support Vector Machine) linear as prediction algorithm. The motivation case is a study done on real data for a problem of bus trip time prediction. In this study we use three different training sets: all the examples, examples from past days similar to the day where prediction is needed, and examples selected by a CART regression tree. Then, we verify if the CART based example selection approach is appropriate on different regression data sets. The experimental results obtained are promising.
\end{abstract}

\section{Introduction}

The performance of prediction algorithms can be improved by selecting the examples used for training. The improvement of performance may correspond to faster training by reducing the number of examples and/or better predictions by selecting the most informative examples [1].

In this paper we approach the problem of example selection (or instance selection) mainly to improve predictive performance.

In section 2 we present results using three different training sets for $\mu$-SVM with the linear kernel and with different parameter sets. All these experiments were done using bus trip time data. In section 3 we present the results of using CART's leaf node (one of the two example selection techniques described in section 2) on different regression data sets collected from L. Torgo's repository (11]) and compare them with the ones from $\mu$-SVM linear without example selection for different parameter sets. We also compare both algorithms using the corresponding best parameter set with CART and linear regression. In section 4 we discuss the results obtained and in the next section we review related work. We end with a review of the work done and a discussion of the guidelines for future work. 


\section{Example Selection for Bus Trip Time Prediction}

In this section we present results of example selection applied to a real decision support problem of bus trip time prediction. This problem consists in predicting three days ahead the duration of a particular urban bus trip. The predicted trip times are then used to define crew duties and reduce costs in terms of extra time payed.

We present results using $\mu$-Support Vector Machine ([8], 7]) with the linear kernel. Tests were done using data from January 1st 2004 to March 31st 2004, i. e., 2646 trip records. The training is done on a sliding window one month long and the test data is one day long. The lag between the train and test sets is three days long.

The explanatory variables used are: (1) start trip time (in seconds); (2) day type (bank holiday, normal, bridge or tolerance); (3) weekday; and (4) day of the year. The bridge day type corresponds to a day, usually a Monday or a Friday, respectively if a bank holiday falls on a Tuesday or a Thursday, that people take as holiday so people can enjoy a four-day weekend. The tolerance day type is similar to the bridge day type, but, in the tolerance case, this day was declared by the government a day that civil servants can take off. The start trip time and the day of the year are numeric while the other two are symbolic. The target variable is the trip time duration and is a numeric one. The series is irregularly time spaced with 29,5 trips per day in average. See [6] for a more complete description of the bus trip time data set.

On these trip time data, we have tried 3 different methods for example selection:

1. All: use all the examples from the same bus line;

2. Equivalent days (ed): use the examples from the same bus line and from identical past days;

3. Leaf node (ln): use the examples from the same bus line and from the same leaf node of a CART (CART - Classification And Regression Trees [2]).

Equivalent days were defined by visual inspection and using experts knowledge. For each bus line were defined nine groups of equivalent days:

- Day Type $=$ Normal and working days (from Monday to Friday)

- Day Type $=$ Normal and Saturdays

- Sundays

- Day Type = bank holiday and weekday on Monday, Friday

- Day Type = bank holiday and weekday on Tuesday, Thursday

- Day Type = bank holiday and Wednesday

- Day Type = bank holiday and Saturdays

- Day Type $=$ bridge day

- Day Type $=$ tolerance day

The example selection using the CART leaf nodes was done by first obtaining a CART regression tree over the initial training set. To predict the time for a 
new trip, we identify the leaf node of the tree where the new trip would belong and obtain all the elements from this leaf node. The members of the training set are the members from the selected leaf node. We have used the rpart (from Rproject [10]) with the default parameters. We use the mean squared error (mse) as evaluation criterion.

Results for different parameter sets are presented in fig. 1, The parameter sets were obtained by combining the two dimensional parameter space $(C$ and $\mu$ ) where $C$ ranges from $2^{(2 *(-\mathbf{2}))}$ to $2^{(2 * \boldsymbol{6})}$; and $\mu$ ranges from $\mathbf{1} / 10$ to $\mathbf{1 0 / 1 0}$. The numbers in bold face will be referred as $C$ index and $\mu$ index, respectively.

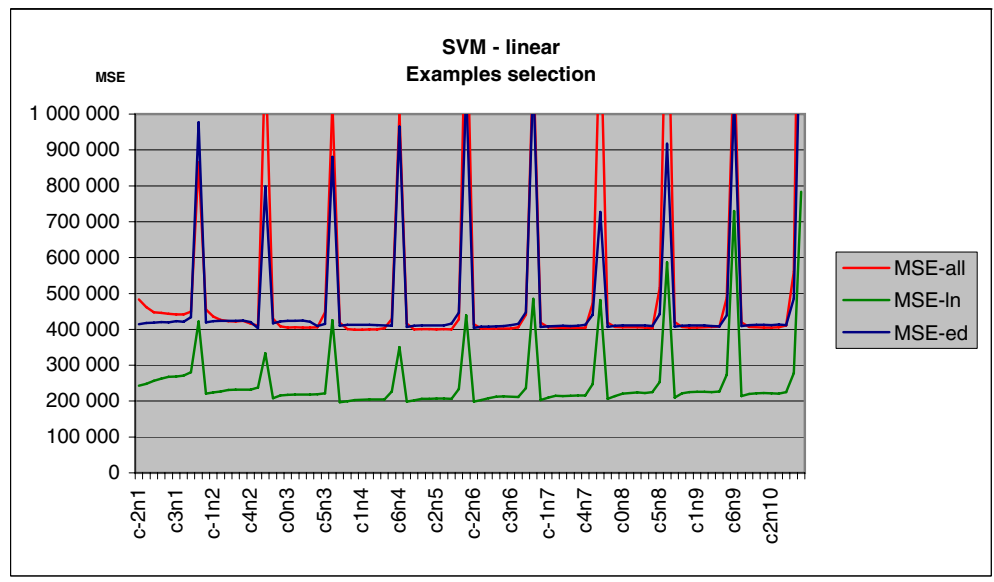

Fig. 1. Example selection for $\mu$-SVM linear. The x-axis's values are expressed as $c[C$ index $] n[\mu$ index $]$.

From the analysis of the results we can see that the best results for all parameter sets are obtained using the leaf node method. These results were the motivation for the study that we describe in the next section.

\section{Using CART's Leaf Node Members as Training Set for $\mu$-SVM Linear on Regression Data Sets}

In this section we validate if the positive results, obtained in the previous section for $\mu$-SVM linear using the CART's leaf node approach for example selection, can be generalized to the regression problem. To run these tests we have collected eleven regression data sets from the L. Torgo's repository ([11]). We have selected data sets of diverse sizes, with the largest one, abalone, having more than 4000 examples.

The experimental procedure (figure 2, adapted from [9]) uses 10-fold cross validation. The selection task was performed by the CART's leaf node approach 
described in the previous section. For each fold, the CART runs once. The parameters tuning was performed by varying $C$ from $2^{(2 *(-4))}$ to $2^{(2 * 4)}$; and $\mu$ from $(2 * \mathbf{0}+1) / 10$ to $(2 * \mathbf{4}+1) / 10$. The 45 parameter sets for both training sets (with and without selection) run over the same 10 folds previously generated, i. e., we are generating paired samples. Pre-all and Pre-ln are, each one, a set of mean squared error values, one for each parameter set tested.

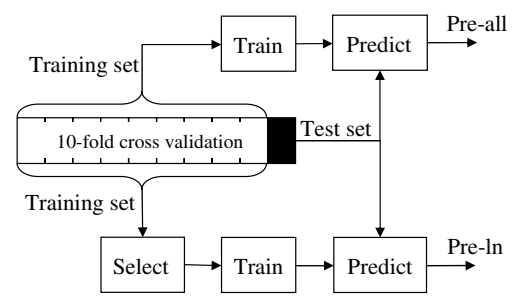

Fig. 2. Experimental procedure

This process was executed 5 times for each data set. In each of the 5 runs, different folds are obtained in 10-fold used for cross-validation. Figures from 3 to 8 present the results.
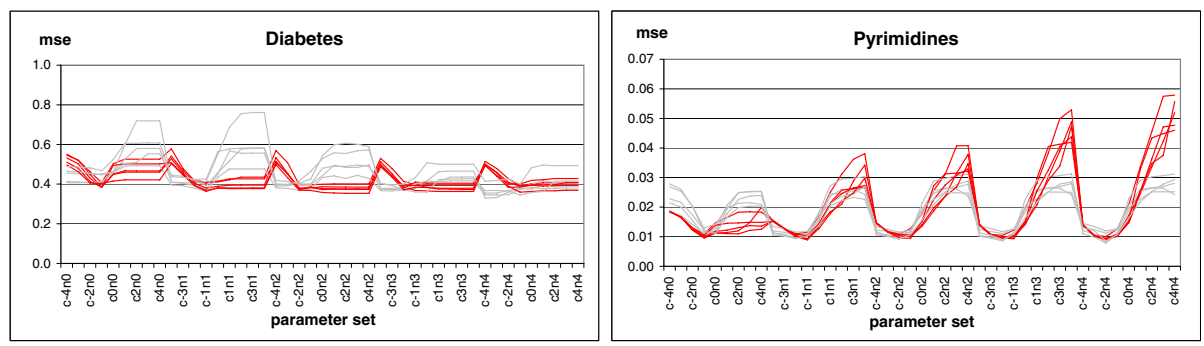

Fig. 3. Diabetes and pyrimidines data sets. The 5 dark lines represent the Pre-all results and the 5 clear lines represent the Pre-ln results. The x-axis's values are expressed as $c[C$ index $] n[\mu$ index $]$.

Figure 9 shows how much relative time is spent using the example selection technique compared with the values obtained using all data. The data sets are ordered by number of examples (ascending order). The values associated to Preall are calculated doing the ratio of the average time to run the 5 Pre-all results over the average time to obtain the 5 Pre-ln results.

Further tests were executed using the parameter set, from the 45 we have tested, with the best average for the 5 observations of Pre-all and the equivalent for Pre-ln. Results using the best parameter set from Pre-all and Pre-ln will be referred as all and ln respectively. We have also compared these results with 

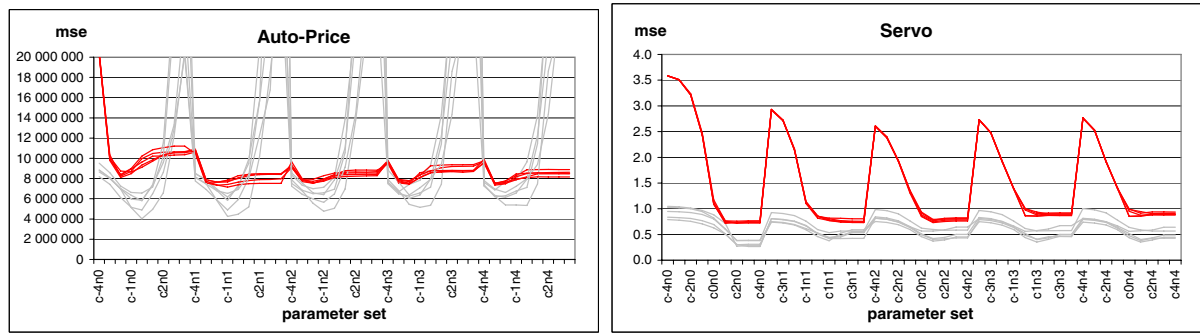

Fig. 4. Auto-price and servo data sets
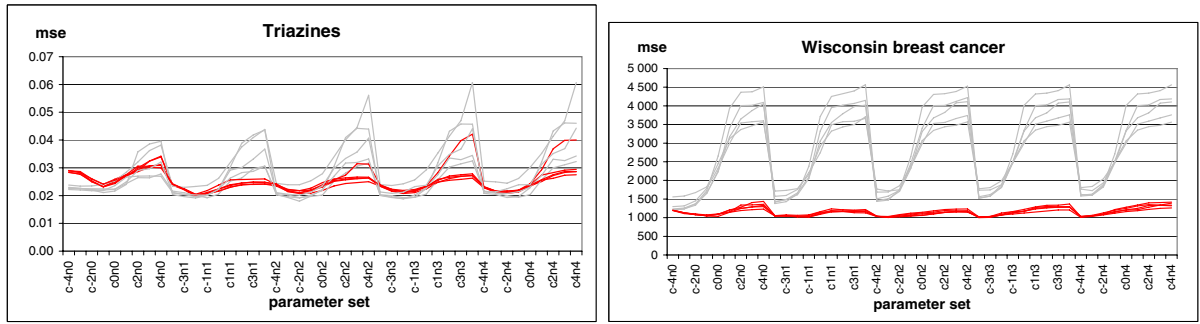

Fig. 5. Triazines and Wisconsin breast cancer datasets
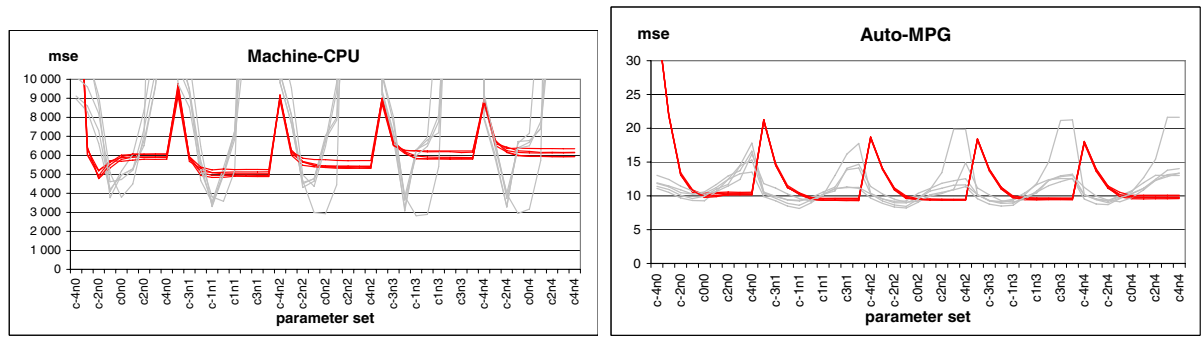

Fig. 6. Machine CPU and auto-MPG data sets
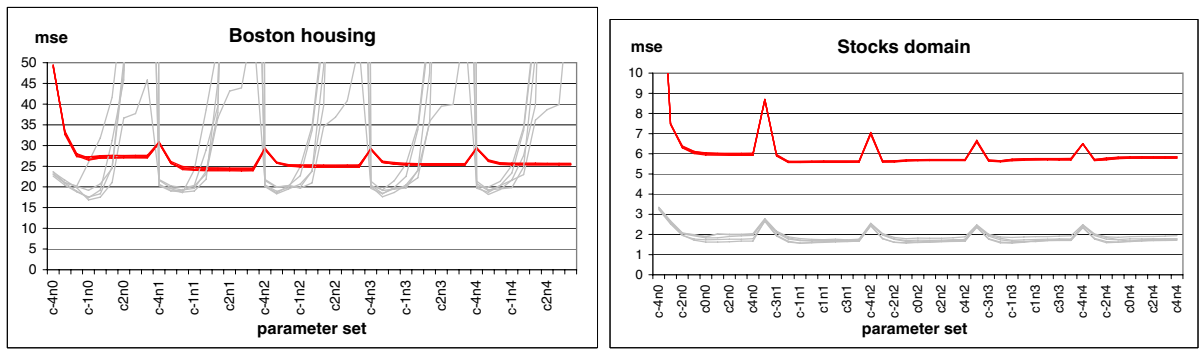

Fig. 7. Boston housing and stocks domain data sets 


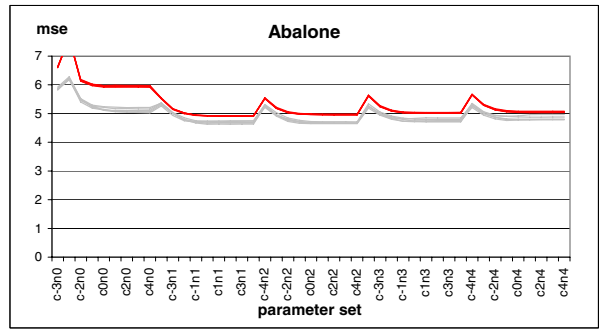

Fig. 8. Abalone data set

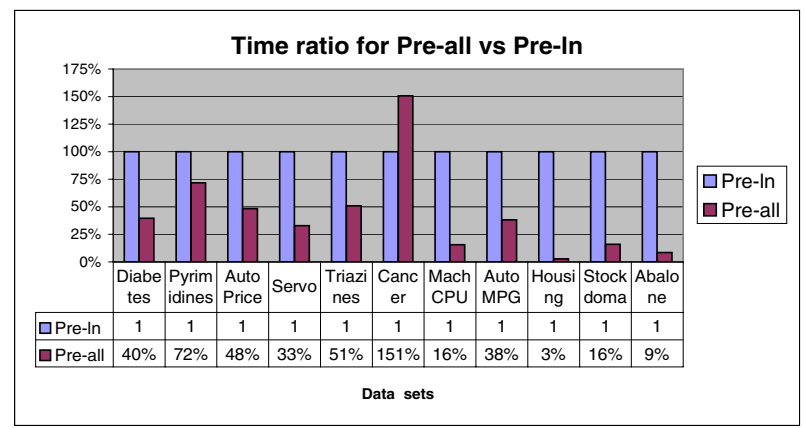

Fig. 9. Time ratio Pre-all vs Pre-ln

CART and linear regression. We got 50 observations for each technique using cross-validation but, this time, the samples are not paired. The lowest mse value for each data set is in bold face. We have used the default parameter set for rpart (CART) and $\mathrm{lm}$ (linear regression) functions from [10] (see figure 10]). Lm results for the pyramidines and triazines data sets are not reported because prediction from a rank-deficient fit may be misleading. Sd represents the standard deviation. The p-value for $\mathrm{lm}$, CART and all refer to the p-value obtained on a hypothesis test about the difference between that technique mean and the mean of the $\ln$ results. The alternate hypothesis is to verify if the $\ln$ mean is lower than the mean of the method being compared. We use a type I error of $5 \%$. The p-value is in bold face when is lower than $5 \%$, i.e, when the null hypothesis is rejected.

Figure 11] compares execution times between the four tested methods.

\section{Discussing Results}

The first tests (presented in figures 3 to 9) intended to tune the parameters in order to select one parameter set for each data set. The tuning was done for two different approaches previously reported (Pre-all and Pre-ln). Some considerations must be done about these tests and their results. 
- In general, results are more sensitive to $\mathrm{C}$ than to $\mu$ parameter values.

- The sensitivity to the parameter sets is very different between data sets and, for some data sets, is also very different between both tested techniques.

- There are several factors affecting time performance results (figure 91). In part, Pre-ln execution time is higher because of CART, even if its complexity is lower than in SVM's case. The use of CART reduces the training examples, reducing the time to train SVM. However, the number of SVM's trained has (if our algorithm was optimized, which is not the case), as upper bound, the number of the CART leaf nodes. In our case we train a new model when the value to predict falls in a different leaf node than the previous value to predict, increasing the number of trained models.

- The time to train a model depends strongly on the parameter set we are using. The higher the values of $\mathrm{C}$ and $\mu$ parameters, the slower is the training time. This is particularly evident in the $\mathrm{C}$ parameter case.

The analysis of results for Pre-all and Pre-ln was done using the Friedman rank sum test since the Kolmogorov-Smirnov Lilliefors test rejects the normality hypothesis and, consequently, it was not possible to use the ANOVA method with just 5 elements for each group. We used as blocks the eleven data sets

\begin{tabular}{|c|c|c|c|c|c|c|c|c|c|c|c|}
\hline & \multicolumn{3}{|c|}{ Im } & \multicolumn{3}{|c|}{ CART } & \multicolumn{3}{|c|}{ all } & \multicolumn{2}{|c|}{ In } \\
\hline Data Set & mean & sd & p-value & mean & sd & p-value & mean & sd & p-value & mean & sd \\
\hline Diabetes & 0.397 & 0.020 & $0.2 \%$ & 0.393 & 0.035 & $2.1 \%$ & 0.384 & 0.019 & $15.7 \%$ & 0.377 & 0.044 \\
\hline Pyrimidines & & & & 0.0122 & 0.00094 & $0.0 \%$ & 0.0093 & 0.00041 & $100.0 \%$ & 0.0101 & 0.00158 \\
\hline Auto Price & 7675516 & 363507 & $0.0 \%$ & 8264533 & 735163 & $0.0 \%$ & 7445161 & 169687 & $0.0 \%$ & 5215136 & 787555 \\
\hline Servo & 0.71 & 0.023 & $0.0 \%$ & 0.77 & 0.073 & $0.0 \%$ & 0.73 & 0.020 & $0.0 \%$ & 0.39 & 0.120 \\
\hline Triazines & & & & 0.0217 & 0.00193 & $0.0 \%$ & 0.0200 & 0.00032 & $21.7 \%$ & 0.0198 & 0.00194 \\
\hline Cancer & 1147 & 41.0 & $100.0 \%$ & 1480 & 94.2 & $0.0 \%$ & 1020 & 12.8 & $100.0 \%$ & 1278 & 71.6 \\
\hline Mach CPU & 4951 & 619 & $0.0 \%$ & 10100 & 1110 & $0.0 \%$ & 5001 & 279 & $0.0 \%$ & 4076 & 1340 \\
\hline Auto MPG & 9.2 & 0.159 & $6.2 \%$ & 11.5 & 0.619 & $0.0 \%$ & 9.4 & 0.159 & $0.0 \%$ & 9.1 & 0.558 \\
\hline Housing & 23.8 & 0.297 & $0.0 \%$ & 23.3 & 1.240 & $0.0 \%$ & 24.5 & 0.389 & $0.0 \%$ & 18.8 & 2.183 \\
\hline Stock domain & 5.51 & 0.023 & $0.0 \%$ & 3.93 & 0.075 & $0.0 \%$ & 5.60 & 0.029 & $0.0 \%$ & 1.64 & 0.126 \\
\hline Abalone & 4.91 & 0.014 & $0.0 \%$ & 5.86 & 0.054 & $0.0 \%$ & 4.91 & 0.011 & $0.0 \%$ & 4.69 & 0.056 \\
\hline
\end{tabular}

Fig. 10. Comparing different algorithms

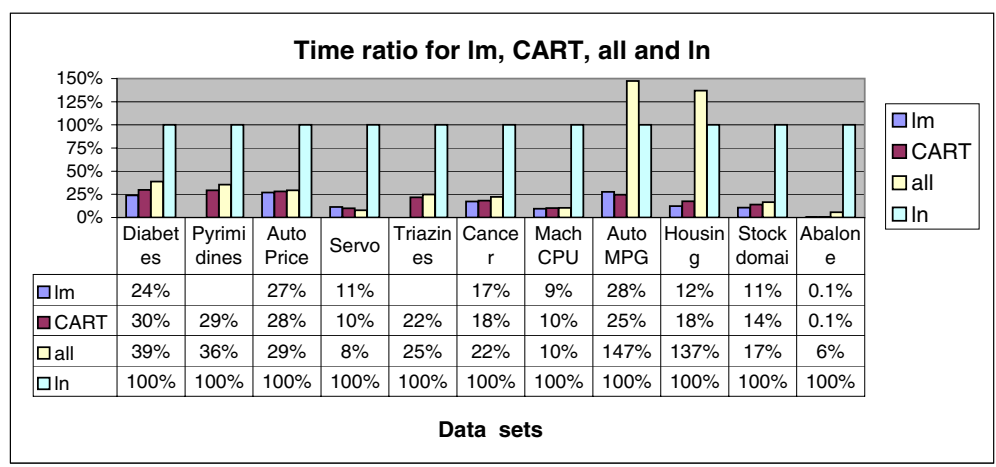

Fig. 11. Time ratio for $1 m$, CART, all and $\ln$ 
Table 1. Friedman rank sum test partial results

\begin{tabular}{|c|c|c|c|c|c|c|c|c|c|c|c|}
\hline Alg ps & $\mathrm{Ri}$ & Alg ps & $\mathrm{Ri}$ & Alg & $\mathrm{Ri}$ & Alg & $\mathrm{Ri}$ & Alg ps & $\mathrm{Ri}$ & Alg & $\mathrm{Ri}$ \\
\hline $\ln c-1 n 1$ & 173 & all c-1n2 & 357 & all c-1n3 & 420 & all c-3n2 & 518 & all c0n0 & 587 & $\ln \quad c 3 n 1$ & 682 \\
\hline $\ln c-2 \mathrm{n} 3$ & 176 & all $c-2 n 2$ & 368 & $\ln \quad c-4 n 3$ & 423 & all $\mathrm{c} 0 \mathrm{n} 4$ & 519 & all $\mathrm{c} 2 \mathrm{n} 4$ & 588 & all $\mathrm{c}-4 \mathrm{n}$ & |083 \\
\hline $\ln \quad c-2 n 4$ & 195 & $\ln c-1 n 0$ & 370 & all c-2n3 & 425 & all c-3n3 & 520 & all $\mathrm{c}-2 \mathrm{n} 0$ & 597 & all $c-4 n$ & 686 \\
\hline $\ln \quad c-2 n 2$ & 197 & all $\mathrm{c} 1 \mathrm{n} 1$ & 380 & all c3n2 & 431 & all $\mathrm{c}-1 \mathrm{n} 0$ & 523 & all c $3 n 4$ & 603 & $\ln \quad \mathrm{c} 3 \mathrm{n} 4$ & 696 \\
\hline $\ln c-1 n 2$ & 210 & all c0n2 & 381 & all c4n2 & 443 & $\ln \quad \mathrm{c} \ln 0$ & 523 & $\ln c-4 n 0$ & 604 & all c- $4 \mathrm{n} 2$ & 700 \\
\hline $\ln \quad c-2 n 1$ & 229 & $\ln \quad c-3 n 1$ & 381 & $\ln \quad \mathrm{c} 0 \mathrm{n} 4$ & 445 & all c2n3 & 531 & all $\mathrm{c} 2 \mathrm{n} 0$ & 605 & c3n3 & 702 \\
\hline $\ln c-1 n 3$ & $247 \mid$ & all c1n2 & 386 & all c-1n4 & 460 & all c3n3 & 536 & all c4n4 & 613 & c3n0 & 706 \\
\hline $\ln \quad \mathrm{c}-1 \mathrm{n} 4$ & 268 & $\ln \quad \mathrm{c} 0 \mathrm{n} 0$ & 392 & $\ln \quad c-4 n 2$ & 462 & all $\mathrm{c} 4 \mathrm{n} 3$ & 549 & $\ln \quad \mathrm{c} 2 \mathrm{n} 1$ & 625 & $\ln \quad \mathrm{c} 4 \mathrm{n} 1$ & 706 \\
\hline $\ln \quad c-3 n 3$ & 293 & all c2n1 & 395 & all $c-2 n 4$ & 465 & all c-3n1 & 550 & $\mathrm{c} 2 \mathrm{n} 2$ & 625 & $\ln \quad \mathrm{c} 4 \mathrm{n} 2$ & 709 \\
\hline all c-1n1 & 297 & $\ln \quad \mathrm{c} 0 \mathrm{n} 2$ & 401 & all c0n3 & 475 & $\ln \quad c \ln 2$ & 555 & all c3no & 627 & $\ln \quad \mathrm{c} 4 \mathrm{n} 4$ & 721 \\
\hline $\ln \quad c-3 n 4$ & 312 & all c3n1 & 402 & $\ln \quad \mathrm{c}-4 \mathrm{n} 1$ & 492 & all c1n4 & 566 & all $\mathrm{c} 4 \mathrm{n} 0$ & 637 & $\ln \quad \mathrm{c} 4 \mathrm{n} 3$ & 727 \\
\hline c0n1 & 322 & all c4n1 & 407 & $\ln c-2 n 0$ & 493 & $\ln \quad c \ln 3$ & 568 & $\mathrm{c} 2 \mathrm{n} 0$ & 646 & all c-4n1 & 729 \\
\hline $\ln c-3 n 2$ & 330 & $\ln \quad \mathrm{c} 0 \mathrm{n} 3$ & 413 & all c1n3 & 500 & $\ln c-3 n$ & 569 & $\mathrm{c} 2 \mathrm{n} 3$ & 646 & $\ln \quad \mathrm{c} 4 \mathrm{n} 0$ & 740 \\
\hline all c0n1 & 345 & $\ln \quad c-4 n 4$ & 416 & $\ln \mathrm{c} \ln 1$ & 508 & all c1n0 & 582 & $\ln \quad \mathrm{c} 2 \mathrm{n} 4$ & 650 & all $c-3 n 0$ & 746 \\
\hline all c-2n1 & 357 & all $\mathrm{c} 2 \mathrm{n} 2$ & 417 & all c-3n4 & 516 & $\ln \quad \mathrm{c} 1 \mathrm{n} 4$ & 584 & $\ln \quad \mathrm{c} 3 \mathrm{n} 2$ & 674 & all $\mathrm{c}-4 \mathrm{n} 0$ & 817 \\
\hline
\end{tabular}

tested, and as groups the combination of the algorithm and the parameter set used ( 2 algorithms $\times 45$ parameter sets). As observation we used the average of the 5 existing observations in each group. The ranking table is presented in table 1 where $\mathrm{Ri}$ is the rank sum for the 11 data sets. The null hypothesis for this test is that the group effect is equal for all the groups. The null hypothesis is rejected with $\mathrm{p}$ value $=0.00 \%$. This result allows us to say that there are meaningful statistical differences between the different algorithms / parameter sets.

We also observe, in Table 1, that the 9 top ranking models were obtained using the $\ln$ approach. It is also a tendency (although with exceptions) that for the same set of parameters, the model obtained with the ln example selection strategy ranks higher than the model obtained with the all strategy.

We have then compared results of four different algorithms: the two previously discussed with the best parameter set pre-selected for each data set, the CART and the linear regression. Some considerations must be done about these tests and their results.

- Time execution for $\ln$ and all (figure 11) are not equivalent to previous results (see figure 9) for all data sets as, for example, the Wisconsin breast cancer, auto-MPG and Boston housing data sets. This happens when the best parameter set for each one of the methods are very different, in particular for the $\mathrm{C}$ parameter. As we have previously said, the execution time increases strongly for higher parameter values. This is particularly true for the $\mathrm{C}$ parameter.

- With the exception of the pyrimidines and the Wisconsin breast cancer data sets, the best results using CART's leaf node members as training set are similar or better than without example selection. 
- For the data sets auto-price, servo, machine-CPU, Boston housing, stocks domain and abalone results using example selection (ln) are meaningfully better compared to all other techniques. For the diabetes and triazines data sets the results are meaningfully better for ln comparing to all other techniques except for $\mu$-SVM linear without example selection (p values of $15.7 \%$ and $21.7 \%$, respectively). For the auto-MPG data set the ln method is not statistically better than the linear model ( $\mathrm{p}$ value of $6.2 \%$ ) but it is for the other two methods (all and CART). Ln performs badly for the Wisconsin breast cancer data set (looses for the all and the linear models) and also for the pyrimidines data set (looses for the all method).

- Ln performs always better than CART. This can be explained by the fact that, in spite of the similarity of the approaches, the prediction model is the average in CART's case and the $\mu$-SVM linear in ln's case (as expected, $\mu$-SVM linear performs better than the average for all data sets).

- Ln variance is much higher than all variance. This can be explained by CART induction algorithm (compares standard deviation values between ln and CART) and also by the reduction of training data in ln method.

\section{Related Work}

In this paper we present a study on example selection in order to improve predictive accuracy $([1])$. Other authors use example selection in order to reduce training time ([5]). The works on example selection and feature selection have many aspects in common ([1]). Techniques for feature selection are usually classified as filters or wrappers (4]). Filter techniques select the features independently of the used induction algorithm while the wrapper approaches have an embedded evaluation of the selection according to the used induction algorithm (4]). Using this classification we can say that our approach is a filter one. A similar approach had been tried before in 9. In that work, the motivation was to test if the use of the support vectors from SVM as training set is an example selection technique that is model independent, i. e., if it can improve results independently of the prediction algorithm chosen. The results obtained in 9, however, are not as expected, since none of the used algorithms (multi-layer perceptrons, nearest neighbors and the C4.5 algorithm for decision trees) improves predictions. Moreover, different algorithms obtain different results. We believe that filter techniques are not model independent, i.e., there is no guarantee that the best training set for a certain induction algorithm is the best one for another induction algorithm. However, we believe that a wrapper approach may be model independent. The wrapper approach has the additional advantage of giving better results than the filter approach. It has the disadvantage of being computationally heavier. Both filter and wrapper approaches are worth researching in the future.

The use of decision trees for feature selection is known ([3]), but we do not know any work using decision trees for example selection. 


\section{Conclusions and Future Work}

This paper describes a study on example selection for $\mu$-SVM linear. Firstly we describe two approaches for example selection on a bus trip time prediction task. We have done tests using $\mu$-SVM with the linear kernel. We observed the impact of example selection for this data set. Then, we tried using as training examples for $\mu$-SVM linear the ones on the same CART's leaf node as the case being predicted. This technique was then tested on other 11 regression data sets. Results show that, with the exception of two data sets, all the others give equal or lower mean squared error when using the example selection technique. This is a very promising result.

Future work will include the reduction of execution time by optimizing our algorithm and by testing faster decision trees induction algorithms, the repetition of these tests for other SVM kernels (we also got promising results using $\mu$-SVM with the gaussian and the sigmoidal kernels for the bus trip time data set), and the tuning of the decision tree induction algorithm parameters.

\section{Acknowledgments}

This work was partially supported by FCT - Fundação para a Ciência e a Tecnologia, project reference: POCT/TRA/61001/2004 and FEDER e Programa de Financiamento Plurianual de Unidades de I\&D.

\section{References}

1. A. L. Blum and P. Langley. Selection of relevant features and examples in machine learning. Artificial Intelligence, 97(1-2):245-271, 1997.

2. L. Breiman, J. H. Friedman, R. A. Olshen, and C. J. Stone. Classification and regression trees. Chapman and Hall/CRC, 1984.

3. C. Cardie. Using decision trees to improve case-based learning. In 10th International conference on machine learning, pages 25-32. Morgan Kaufmann, 1993.

4. R. Kohavi and G. H. John. Wrappers for feature subset selection. Artificial Intelligence, 97(1-2):273-324, 1997.

5. H. Liu and H. Motoda. On issues of instance selection. Data Mining and Knowledge Discovery, 6(2):115-130, 2002.

6. J. M. Moreira, A. Jorge, J. F. Sousa, and C. Soares. Trip time prediction in mass transit companies. a machine learning approach. In 10th EWGT, pages 276-283, 2005 .

7. B. Scholkopf, A. J. Smola, R. Williamson, and P. Bartlett. New support vector algorithms. Technical Report NC2-TR-1998-031, 1998.

8. A. J. Smola and B. Scholkopf. A tutorial on support vector regression. Technical Report NC2-TR-1998-030, 1998.

9. N. A. Syed, H. Liu, and K. K. Sung. A study of support vectors on model independent example selection. In 5th ACM SIGKDD, pages 272-276, 1999.

10. R. D. C. Team. R: A language and environment for statistical computing. Technical report, R Foundation for Statistical Computing, 2004.

11. L. Torgo. Regression data repository, http://www.liacc.up.pt/ ltorgo. 